\title{
Effect of Prior Biologic Use on Cost-Effectiveness of Brodalumab vs. Ustekinumab for Treatment of Moderate-to-Severe Psoriasis in the United States
}

Steven R. Feldman · Shipra Rastogi · Jay Lin

Received: June 5, 2018 / Published online: July 13, 2018

(C) The Author(s) 2018

\section{ABSTRACT}

Introduction: Clinical trials have shown brodalumab to have better efficacy than ustekinumab for the treatment of moderate-to-severe psoriasis. An estimation of the cost-effectiveness of brodalumab vs. ustekinumab is warranted and may be useful for treatment decision-making processes, especially in the context of the cost considerations of the current US healthcare system. Therefore, we compared the cost-effectiveness of brodalumab with ustekinumab for treatment of moderate-to-severe psoriasis in biologic-naïve and biologic-experienced patients in the USA.

Methods: An Excel-based economic model was developed to estimate and compare total annual costs to health plans associated with

Enhanced digital features To view enhanced digital features for this article go to https://doi.org/10.6084/ m9.figshare.6741239.

S. R. Feldman $(\square)$

Wake Forest University School of Medicine, Winston-Salem, NC, USA

e-mail: sfeldman@wakehealth.edu

S. Rastogi

Ortho Dermatologics, Bridgewater, NJ, USA

J. Lin

Novosys Health, Green Brook, NJ, USA treatment with brodalumab vs. ustekinumab per achievement of Psorasis Area and Severity Index (PASI) 75, 90, and 100 for patients with moderate-to-severe psoriasis.

Results: For treatment with brodalumab vs. ustekinumab, total annual treatment costs per PASI 75,90 , and 100 were $\$ 31,106, \$ 57,776$, and $\$ 163,069$, respectively, lower for a patient naïve to prior biologic treatment; they were $\$ 40,535, \$ 65,472$, and $\$ 223,610$, respectively, lower for a patient experienced with prior biologic treatment. In an additional analysis among patients with and without prior biologic failure, they were $\$ 52,822, \$ 93,309$, and $\$ 365,606$, respectively, lower for a patient with failure and they were $\$ 31,660, \$ 57,128$, and $\$ 164,996$, respectively, lower for a patient without failure.

Conclusion: Compared to ustekinumab, treatment with brodalumab was associated with better cost-effectiveness ratios for biologic-naïve and experienced-patients and also patients with and without prior biologic treatment failure. The greater cost-effectiveness of brodalumab was most prominent for biologic-experienced and prior biologic treatment failure patients.

Funding: Ortho Dermatologics.

Keywords: Biologics; Brodalumab; Costeffectiveness; Psoriasis; Ustekinumab 


\section{INTRODUCTION}

Psoriasis is a chronic systemic inflammatory disease that primarily affects the skin; it is also associated with an increased risk for several serious comorbidities, including psoriatic arthritis, inflammatory bowel disease, cardiovascular disease, diabetes, cancer, and depression [1]. Psoriasis affects as many as 7.5 million individuals in the USA, with $5 \%$ to $17 \%$ of them having moderate-to-severe disease $[2,3]$. In the USA in 2013 the annual indirect and direct cost of psoriasis was reported to be $\$ 112$ billion [4].

With greater understanding of the immunopathogenesis of psoriasis, several targeted monoclonal antibodies have been developed in the last decade that have demonstrated remarkable efficacy in clinical trials for the treatment of moderate-to-severe psoriasis $[5,6]$. Ustekinumab was the first monoclonal antibody targeting the interleukin (IL)-12/-23 pathway to be approved by the Food and Drug Administration (FDA) in the USA (2009) for the treatment of moderate-to-severe psoriasis [7]. Brodalumab was approved by the FDA in February of 2017 [8]. It is a human monoclonal antibody that selectively targets the IL-17 receptor $\mathrm{A}$, thereby blocking pro-inflammatory downstream signaling $[5,6]$.

The results of two phase III clinical trials, AMAGINE-2 and AMAGINE-3, showed that brodalumab had superior efficacy over ustekinumab after 12 weeks of treatment for achieving Psorasis Area and Severity Index (PASI) 75, 90 , and 100 ( $85.7 \%$ vs. $69.7 \%, 69.5 \%$ vs. $47.3 \%$, and $40.5 \%$ vs. $20.1 \%$ of patients, respectively, all $p<0.001$ ) [9]. A recently published integrated analysis of the AMAGINE-2/-3 trials reported that the superior efficacy of brodalumab compared to ustekinumab was observed among both patients naïve to biologic treatment (PASI 75: $87.1 \%$ vs. $72.2 \%$; PASI 90: $71.6 \%$ vs. $48.7 \%$; PASI 100 : $40.9 \%$ vs. $21.1 \%$ ) and patients experienced with biologic treatment (PASI 75: $81.7 \%$ vs. $62.3 \%$; PASI 90: $63.8 \%$ vs. $43.4 \%$; PASI 100 : $39.5 \%$ vs. $17.0 \%$ ) [10]. These results were also true among both patients with prior biologic treatment failure (PASI 75: $81.3 \%$ vs. $54.8 \%$; PASI 90: $62.7 \%$ vs.
35.5\%; PASI 100: $32.0 \%$ vs. $11.3 \%$ ) and patients without prior biologic treatment failure (PASI 75: $86.3 \%$ vs. $71.3 \%$; PASI 90 : $70.4 \%$ vs. $48.6 \%$; PASI 100: $41.7 \%$ vs. $21.1 \%$ ) [10].

The better efficacy of brodalumab vs. ustekinumab across different patient types [10] suggests that it may be a good treatment option among not only patients initiating biologic therapy but also those who have had prior biologic exposure and may want to switch to another biologic drug. An estimation of the cost-effectiveness of brodalumab vs. ustekinumab across such patient types is warranted and may be useful for treatment decision-making processes, especially in the context of the cost considerations of the current US healthcare system. Therefore, we used the efficacy data provided in the recent integrated analysis of the AMAGINE-2/-3 clinical trials to compare the expected cost-effectiveness of brodalumab with ustekinumab for the treatment of patients with moderate-to-severe psoriasis with and without prior exposure to a biologic and with and without prior biologic treatment failure.

\section{METHODS}

\section{Description of Economic Model}

A cost-effectiveness economic model from a US payer perspective was constructed in Excel to compare the average annual cost to health plans for achieving PASI 75, PASI 90, and PASI 100 among moderate-to-severe psoriasis patients treated with brodalumab (Siliq ${ }^{\mathrm{TM}}$, Ortho Dermatologics, Bridgewater, NJ) or ustekinumab (Stelara ${ }^{\circledR}$, Janssen Biotech, South Raritan, NJ). This economic model is based on previously conducted studies and does not contain any input with human participants or animals performed by any of the authors.

\section{Model Inputs}

PASI 75, PASI 90, and PASI 100 efficacy data for brodalumab and ustekinumab were obtained from the published analysis results of the AMAGINE-2 and AMAGINE-3 clinical trials in 
moderate-to-severe psoriasis patients [10]. The 2018-year wholesale acquisition cost (WAC) data of the biologics were obtained from RedBook [11]. Drug dispensing fee [12, 13], patient co-pay [12, 13], and drug contracting discount (a hypothetical estimate of $20 \%$ ) were incorporated into the model [13]. The average annual total medical costs associated with a non-response to biologic treatment $(\mathrm{PASI}<75)$ and potential adverse event (AE) monitoring (initial and every 3 months) were derived from published literature and also incorporated into the model [14]. Model inputs for a patient naïve to prior biologic treatment and a patient experienced with prior biologic treatment are shown in Table 1 and inputs for a patient with and without prior biologic treatment failure are shown in Table 2.

\section{Estimation of Cost-Effectiveness of Brodalumab vs. Ustekinumab Treatment to US Health Plans}

The total annual costs to health plans for treatment with brodalumab and ustekinumab for a patient naïve to prior biologic treatment and a patient experienced with prior biologic treatment were estimated. An additional analysis was conducted for a patient with prior biologic treatment failure and a patient without prior biologic treatment failure. Cost-effectiveness of brodalumab and ustekinumab were compared for the different patient types using the estimated average treatment cost per achievement of PASI 75, 90, and 100.

\section{Sensitivity Analyses}

Univariate (one-way) sensitivity analyses were conducted to determine the effects of varying a single model parameter at a time on the estimated differences in total annual health plan costs per PASI 75 for each patient type. The model parameters that were varied in the univariate analyses included brodalumab and ustekinumab PASI 75 efficacy, drug cost discount, patient co-pay, medical cost associated with non-responder (PASI $<75$ ), and biologic drug AE monitoring costs. The impact of the variations of such model parameters on the cost differences of ustekinumab vs. brodalumab for achievement of PASI 75 (cost per PASI 75) were estimated when the model parameters were varied between the ranges of their respective $95 \%$ confidence intervals. When the confidence intervals were unknown, such as for the cost parameters, they were varied $\pm 30 \%$.

Since in the real-world settings, the model parameters may often be interdependent, Monte Carlo multivariable sensitivity analyses were also conducted. For each cycle of the Monte Carlo analyses, the value of the PASI efficacy rate was taken randomly from a Gaussian distribution of the mean and standard deviation of the corresponding variables. When the standard deviations were unknown, such as for the cost parameters, a coefficient of variation of $\pm 30 \%$ is used for the Gaussian distribution. Ten thousand such iterations were conducted for each biologic comparison. Descriptive statistics of the total cost differences were reported from the results of the 10,000 such random Monte Carlo cycles for each cost per PASI comparison. The 95\% confidence intervals (CIs) of the mean cost per PASI differences were evaluated as the range between the 2.5 and 97.5 percentile of cost per PASI differences from the 10,000 random cycles of Monte Carlo simulation for ustekinumab vs. brodalumab comparison. To further evaluate the model robustness and result distribution, the Monte Carlo multivariable analysis was carried out for both the assessment of the differences in cost per PASI 75 and cost per PASI 100.

\section{RESULTS}

\section{Estimation of Total Annual Health Plan Costs for Treatment with Brodalumab and Ustekinumab per Patient}

The 2018 WACs for brodalumab and ustekinumab were $\$ 1750$ and $\$ 13,180$, respectively [11]. The number of injections per year was based on prescribing information and was estimated at 27 injections per year for brodalumab and 5 injections per year for ustekinumab $[7,8]$. The 45/90 mg dosage mix for ustekinumab used 
Table 1 Key model inputs for moderate-to-severe psoriasis patients naïve to and experienced with prior biologic treatment

\begin{tabular}{|c|c|c|c|c|}
\hline & \multicolumn{2}{|l|}{ Biologic-naïve } & \multicolumn{2}{|c|}{ Biologic-experienced } \\
\hline & Brodalumab & Ustekinumab & Brodalumab & Ustekinumab \\
\hline Drug dosage for initiation and maintenance & $210 \mathrm{mg}$ & $45 / 90 \mathrm{mg} \operatorname{mix}$ & $210 \mathrm{mg}$ & $45 / 90 \mathrm{mg} \operatorname{mix}$ \\
\hline \multicolumn{5}{|l|}{ Drug efficacy $^{a}[10]$} \\
\hline PASI 75 (95\% CI) & $\begin{array}{l}87.1 \% \\
\quad(84.8-89.3 \%)\end{array}$ & $\begin{array}{l}72.2 \% \\
\quad(67.9-76.3 \%)\end{array}$ & $\begin{array}{l}81.7 \% \\
\quad(77.2-85.7 \%)\end{array}$ & $\begin{array}{l}62.3 \% \\
\quad(54.2-69.8 \%)\end{array}$ \\
\hline PASI $90(95 \%$ CI $)$ & $\begin{array}{l}71.6 \% \\
\quad(68.6-74.5 \%)\end{array}$ & $\begin{array}{l}48.7 \% \\
\quad(44.0-53.4 \%)\end{array}$ & $\begin{array}{l}63.8 \% \\
\quad(58.4-68.9 \%)\end{array}$ & $\begin{array}{l}43.4 \% \\
\quad(35.6-51.5 \%)\end{array}$ \\
\hline PASI 100 (95\% CI) & $\begin{array}{l}40.9 \% \\
\quad(37.7-44.2 \%)\end{array}$ & $\begin{array}{l}21.1 \% \\
\quad(17.5-25.2 \%)\end{array}$ & $\begin{array}{l}39.5 \% \\
\quad(34.2-45.0 \%)\end{array}$ & $\begin{array}{l}17.0 \% \\
\quad(11.5-23.7 \%)\end{array}$ \\
\hline \multicolumn{5}{|l|}{ Drug costs } \\
\hline Drug dispensing fee per $\mathrm{Rx}[12,13]$ & $\$ 3$ & $\$ 3$ & $\$ 3$ & $\$ 3$ \\
\hline Average patient co-pay per $\mathrm{Rx}[12,13]$ & $\$ 51$ & $\$ 51$ & $\$ 51$ & $\$ 51$ \\
\hline Drug cost without discount per patient-year & $\$ 47,250$ & $\$ 65,900$ & $\$ 47,250$ & $\$ 65,900$ \\
\hline Hypothetical drug discount level & $20 \%$ & $20 \%$ & $20 \%$ & $20 \%$ \\
\hline Drug cost after discount per patient-year & $\$ 37,800$ & $\$ 52,720$ & $\$ 37,800$ & $\$ 52,720$ \\
\hline Total patient payment per patient-year & $\$ 612$ & $\$ 255$ & $\$ 612$ & $\$ 255$ \\
\hline Total health plan drug payment per patient-year & $\$ 37,224$ & $\$ 52,480$ & $\$ 37,224$ & $\$ 52,480$ \\
\hline $\begin{array}{l}\text { Annual medical cost associated with psoriasis } \\
\text { non-response }(\text { PASI }<75)[14]\end{array}$ & $\$ 670$ & $\$ 1342$ & $\$ 914$ & $\$ 1789$ \\
\hline Annual AE monitoring $\operatorname{cost}^{\mathrm{b}}[14]$ & $\$ 278$ & $\$ 278$ & $\$ 278$ & $\$ 278$ \\
\hline Total annual cost per patient to health plan & $\$ 38,172$ & $\$ 54,100$ & $\$ 38,416$ & $\$ 54,547$ \\
\hline
\end{tabular}

$P A S I$ Psoriasis Area and Severity Index, $C I$ confidence interval, $R x$ prescription, $A E$ adverse event

a Assessed at 12 weeks of treatment [10]

b Includes initial monitoring and monitoring every 3 months

in the model was based on the data of the AMAGINE-2/-3 trials, in which $72 \%$ of patients with moderate-to-severe psoriasis used the $45 \mathrm{mg}$ dosage of ustekinumab [10]. After incorporating the annual drug cost (with patient copay and dispensing fee per prescription included and a $20 \%$ hypothetical discount applied), medical cost associated with psoriasis non-response, and $\mathrm{AE}$ monitoring costs, the total annual costs to a health plan for treatment with brodalumab were estimated at $\$ 38,172$ for a patient naïve to prior biologic treatment, $\$ 38,146$ for a patient experienced with prior biologic treatment (Table 1), $\$ 38,434$ for a patient with prior biologic treatment failure, and $\$ 38,208$ for a patient without prior biologic treatment failure (Table 2). The total annual costs to a health plan for treatment with ustekinumab were estimated at $\$ 54,100$ for a patient naïve to prior biologic treatment, $\$ 54,547$ for a patient experienced with prior biologic treatment (Table 1), $\$ 54,885$ for a patient with prior biologic treatment failure, and $\$ 54,141$ for a patient without prior biologic treatment failure (Table 2). These estimated annual health plan costs were driven mostly by 
Table 2 Key model inputs for moderate-to-severe psoriasis patients with and without prior biologic treatment failure

\begin{tabular}{|c|c|c|c|c|}
\hline & \multicolumn{2}{|c|}{ With biologic failure } & \multicolumn{2}{|c|}{ Without biologic failure } \\
\hline & Brodalumab & Ustekinumab & Brodalumab & Ustekinumab \\
\hline Drug dosage for initiation and maintenance & $210 \mathrm{mg}$ & 45/90 mg mix & $210 \mathrm{mg}$ & $45 / 90 \mathrm{mg} \operatorname{mix}$ \\
\hline \multicolumn{5}{|l|}{ Drug efficacy $^{a}[10]$} \\
\hline PASI 75 (95\% CI) & $\begin{array}{l}81.3 \% \\
\quad(74.2-87.2 \%)\end{array}$ & $\begin{array}{l}54.8 \% \\
\quad(41.7-67.5 \%)\end{array}$ & $\begin{array}{l}86.3 \% \\
\quad(84.1-88.3 \%)\end{array}$ & $\begin{array}{l}71.3 \% \\
\quad(67.4-75.1 \%)\end{array}$ \\
\hline PASI 90 (95\% CI) & $\begin{array}{l}62.7 \% \\
\quad(54.4-70.4 \%)\end{array}$ & $\begin{array}{l}35.5 \% \\
\quad(23.7-48.7 \%)\end{array}$ & $\begin{array}{l}70.4 \% \\
\quad(67.6-73.1 \%)\end{array}$ & $\begin{array}{l}48.6 \% \\
\quad(44.4-52.9 \%)\end{array}$ \\
\hline PASI $100(95 \% \mathrm{CI})$ & $\begin{array}{l}32.0 \% \\
\quad(24.6-40.1 \%)\end{array}$ & $\begin{array}{l}11.3 \% \\
\quad(4.7-21.9 \%)\end{array}$ & $\begin{array}{l}41.7 \% \\
\quad(38.8-44.7 \%)\end{array}$ & $\begin{array}{l}21.1 \% \\
\quad(17.7-24.7 \%)\end{array}$ \\
\hline \multicolumn{5}{|l|}{ Drug costs } \\
\hline Drug dispensing fee per $\mathrm{Rx}[12,13]$ & $\$ 3$ & $\$ 3$ & $\$ 3$ & $\$ 3$ \\
\hline Average patient co-pay per $\mathrm{Rx}[12,13]$ & $\$ 51$ & $\$ 51$ & $\$ 51$ & $\$ 51$ \\
\hline Drug cost without discount per patient-year & $\$ 47,250$ & $\$ 65,900$ & $\$ 47,250$ & $\$ 65,900$ \\
\hline Hypothetical drug discount level & $20 \%$ & $20 \%$ & $20 \%$ & $20 \%$ \\
\hline Drug cost after discount per patient-year & $\$ 37,800$ & $\$ 52,720$ & $\$ 37,800$ & $\$ 52,720$ \\
\hline Total patient payment per patient-year & $\$ 612$ & $\$ 255$ & $\$ 612$ & $\$ 255$ \\
\hline Total health plan drug payment per patient-year & $\$ 37,224$ & $\$ 52,480$ & $\$ 37,224$ & $\$ 52,480$ \\
\hline $\begin{array}{l}\text { Annual medical cost associated with psoriasis } \\
\text { non-response }(\text { PASI }<75)[14]\end{array}$ & $\$ 932$ & $\$ 2127$ & $\$ 706$ & $\$ 1383$ \\
\hline Annual AE monitoring $\operatorname{cost}^{\mathrm{b}}[14]$ & $\$ 278$ & $\$ 278$ & $\$ 278$ & $\$ 278$ \\
\hline Total annual cost per patient to health plan & $\$ 38,434$ & $\$ 54,885$ & $\$ 38,208$ & $\$ 54,141$ \\
\hline
\end{tabular}

$P A S I$ Psoriasis Area and Severity Index, $C I$ confidence interval, $R x$ prescription, $A E$ adverse event

${ }^{a}$ Assessed at 12 weeks of treatment [10]

b Includes initial monitoring and monitoring every 3 months

annual drug WAC and different PASI 75 response levels achieved among different patient populations.

\section{Estimation of Total Annual Health Plan Costs for Treatment with Brodalumab vs. Ustekinumab per PASI 75, 90, and 100}

On the basis of this economic model, the total annual treatment costs of a moderate-to-severe psoriasis patient naïve to prior biologic treatment were $\$ 31,106$ (42\% lower), $\$ 57,776$ (52\% lower), and $\$ 163,069$ (64\% lower) lower for treatment with brodalumab vs. ustekinumab per PASI 75, 90, and 100, respectively (Fig. 1a). The total annual treatment costs of a patient experienced with prior biologic treatment were $\$ 40,535$ (46\% lower), $\$ 65,472$ (52\% lower), and $\$ 223,610$ (70\% lower) lower for treatment with brodalumab vs. ustekinumab per PASI 75, 90, and 100, respectively (Fig. 1b). The cost reductions associated with brodalumab vs. ustekinumab per PASI 75, 90, and 100 were more substantial for a patient experienced with prior biologic treatment vs. a patient naïve to prior biologic treatment, especially per PASI 100. However, the magnitudes (\% lower) of the cost 

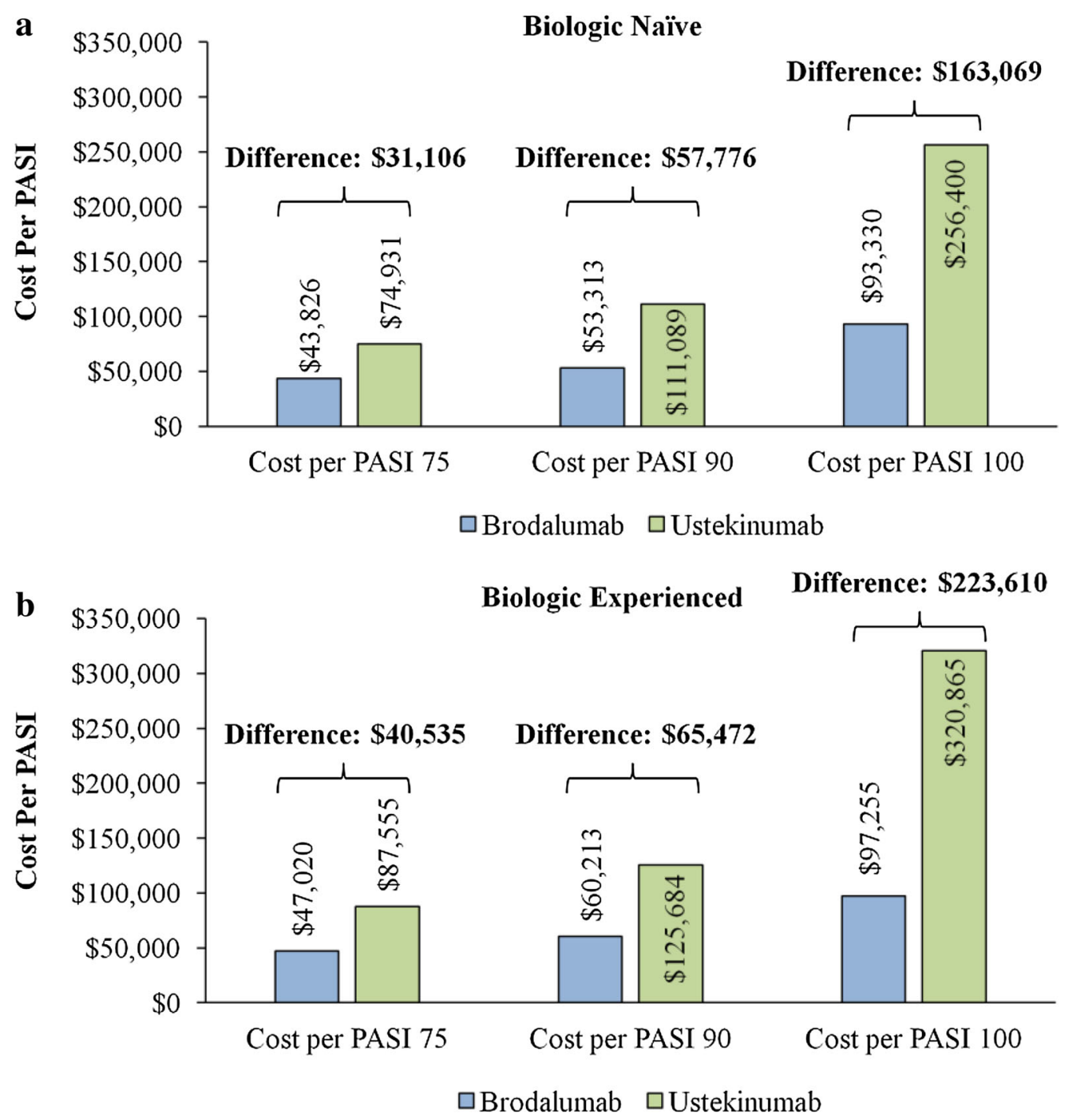

Fig. 1 Total annual health plan costs per PASI 75, 90, and 100 among a biologic-naïve and $\mathbf{b}$ biologic-experienced patients treated with brodalumab and ustekinumab. PASI Psoriasis Area Severity Index

reductions were relatively similar for these patient types.

The total annual treatment costs of a patient with prior biologic treatment failure were $\$ 52,822$ (53\% lower), \$93,309 (60\% lower), and $\$ 365,606$ (75\% lower) lower for treatment with brodalumab vs. ustekinumab per PASI 75, 90, and 100, respectively (Fig. 2a). The total annual treatment costs of a patient without prior biologic treatment failure were \$31,660 (42\% lower), $\$ 57,128$ (51\% lower), and $\$ 164,996$ (64\% lower) lower for treatment with brodalumab vs. ustekinumab per PASI 75, 90, and
100, respectively (Fig. 2b). The cost reductions associated with brodalumab vs. ustekinumab per PASI 75, 90, and 100 were more substantial for a patient with prior biologic treatment failure vs. a patient without prior biologic treatment failure, especially per PASI 100. The magnitude (\% lower) of the cost reductions associated with brodalumab vs. ustekinumab treatment were also greater for a patient with prior biologic treatment failure vs. a patient without prior biologic treatment failure. 
a

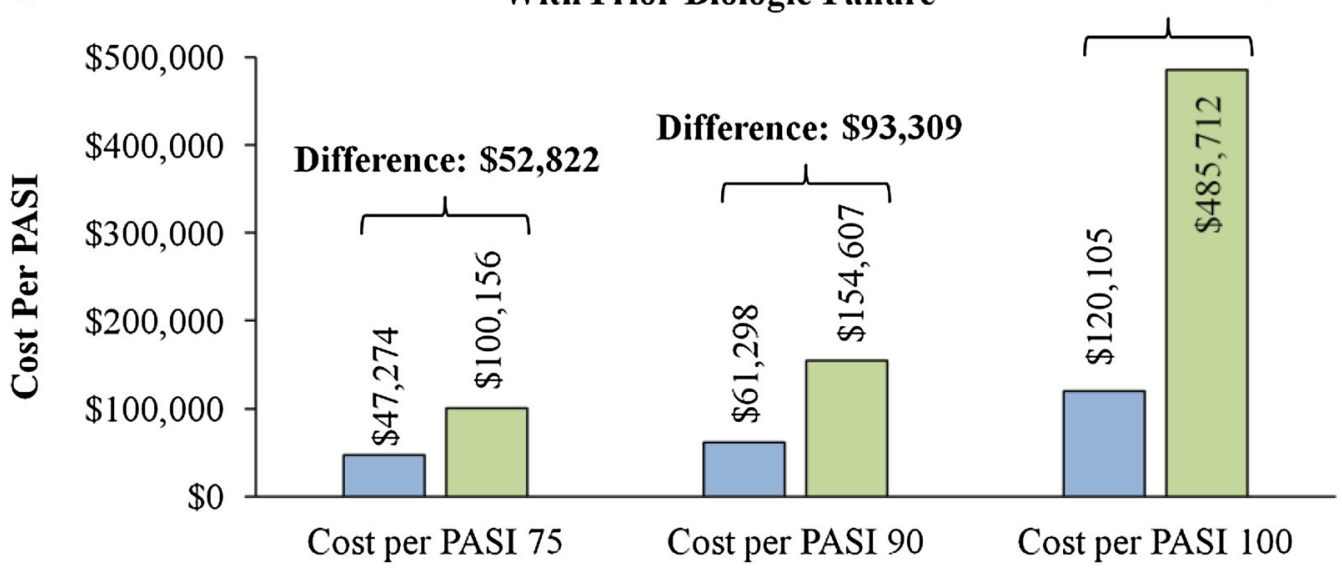

$\square$ Brodalumab $\square$ Ustekinumab b

$$
\$ 500,000
$$

For patients naïve to and experienced with prior biologic treatment, the univariate (Fig. 3) and multivariable (Fig. 4) sensitivity analyses demonstrated consistent differences in annual health plan costs per PASI 75 associated with treatment with ustekinumab vs. brodalumab. Variations in the PASI 75 efficacy rate of ustekinumab had the greatest impact on the cost per PASI 75 differences for both a patient naïve to prior biologic treatment $(+\$ 26,837$ to $+\$ 36,137)$ and a patient experienced with prior biologic treatment $(+\$ 30,642$ to $+\$ 54,294)$. Variations in the PASI 75 efficacy rate of brodalumab and drug cost discount also had relatively large impacts on the cost per PASI 75 differences associated with treatment with ustekinumab vs. brodalumab. Variations in the other model parameters had relatively small impacts on the cost per PASI 75 differences. The variation in the cost per PASI 75 differences was on a larger scale for a patient experienced with prior biologic treatment vs. a biologic-naïve patient. 
The Monte Carlo multivariable analyses, in which each variable of the univariate analysis was allowed to vary simultaneously for 10,000 cycles, was used to further test the consistency of the estimated cost differences per PASI 75 for treatment with ustekinumab vs. brodalumab. The mean cost per PASI 75 difference (with 95\% CI) associated with treatment with ustekinumab vs. brodalumab for a patient naïve to prior biologic treatment was $+\$ 31,151(+\$ 26,109$ to $+\$ 36,765)$ and for a patient experienced with prior biologic treatment it was $+\$ 40,880$ $(\$ 29,568$ to $+\$ 54,154)$. For both biologic-naïve and biologic-experienced patients, in $100 \%$ of the 10,000 random Monte Carlo simulation cycles, ustekinumab displayed a higher cost per PASI 75 vs. brodalumab (100.0\% of Monte Carlo simulation cycles had a cost difference $>\$ 0$ ). The mean cost per PASI difference was magnified for achievement of PASI 100. In this Monte Carlo multivariable analysis the mean cost per

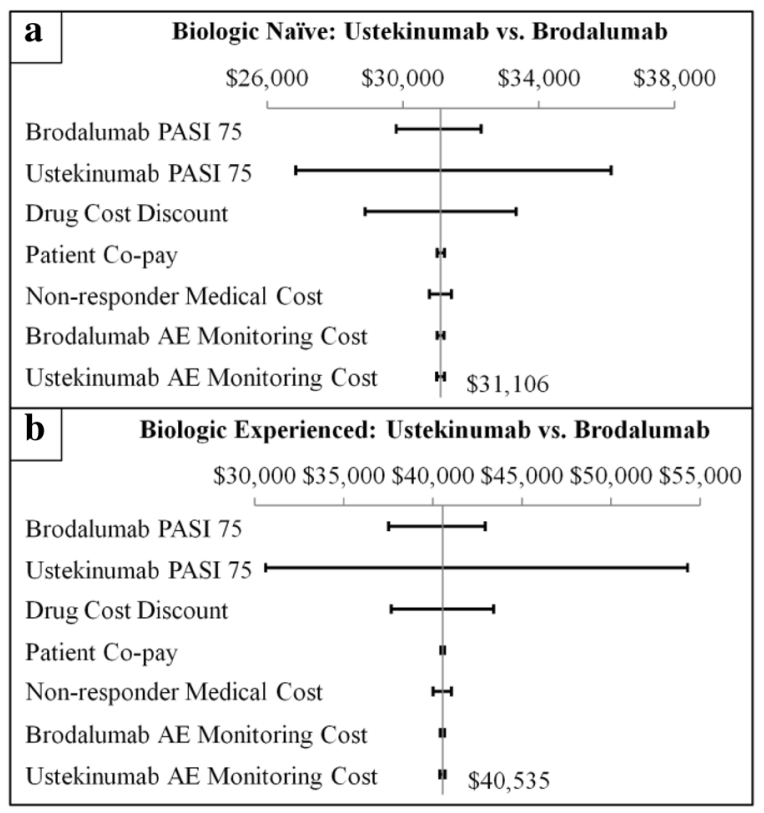

Fig. 3 Univariate sensitivity analysis of the differences in total annual health plan costs per PASI 75 among $\mathbf{a}$ biologic-naïve and $\mathbf{b}$ biologic-experienced patients treated with ustekinumab vs. brodalumab. PASI Psoriasis Area Severity Index. PASI 75 efficacy rates were varied between the ranges of their respective $95 \%$ confidence intervals; incremental cost estimates were varied $\pm 30 \%$. Nonresponder medical cost: average annual total medical costs associated with PASI $<75$. AE adverse event

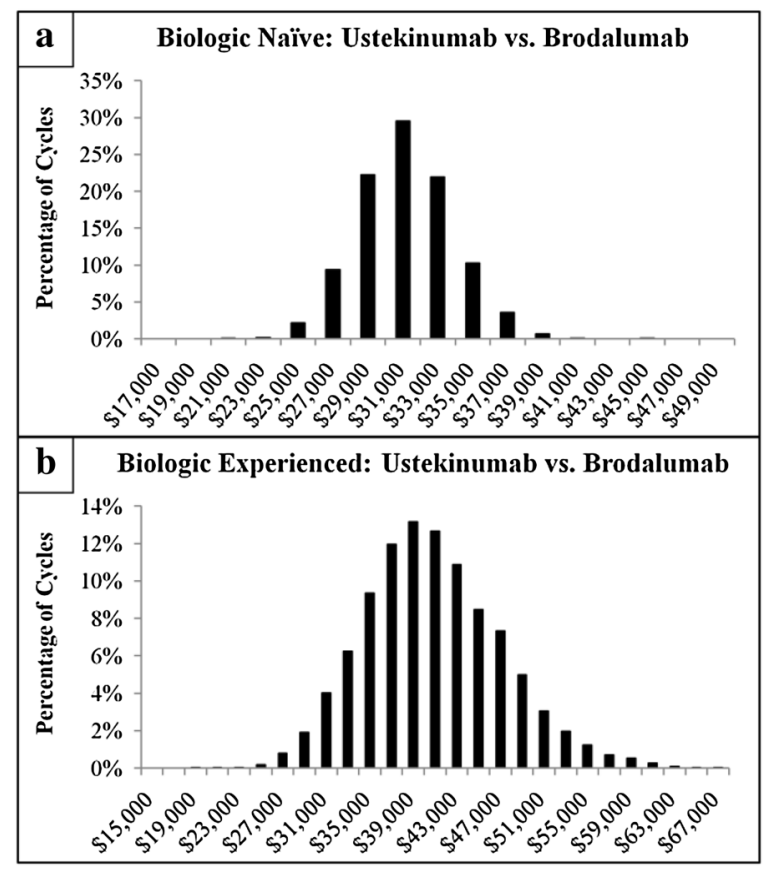

Fig. 4 Multivariable sensitivity analysis of the differences in total annual health plan costs per PASI 75 among $\mathbf{a}$ biologic-naïve and $\mathbf{b}$ biologic-experienced patients treated with ustekinumab vs. brodalumab. PASI Psoriasis Area Severity Index

PASI 100 difference was $+\$ 164,816$ $(+\$ 121,474$ to $+\$ 222,448)$ for a patient naïve to prior biologic treatment and for a patient experienced with prior biologic treatment was $+\$ 234,694(+\$ 137,411$ to $+\$ 400,340)$. For both biologic-naïve and biologic-experienced patients, in $100 \%$ of the 10,000 random Monte Carlo simulation cycles, ustekinumab displayed a higher cost per PASI 100 vs. brodalumab (100.0\% of Monte Carlo simulation cycles had a cost difference $>\$ 0$ ).

For patients with and without prior biologic treatment failure, the univariate (Fig. 5) and multivariable (Fig. 6) sensitivity analyses also demonstrated consistent differences in annual health plan costs per PASI 75 associated with treatment with ustekinumab vs. brodalumab. Variations in the PASI 75 efficacy rate of ustekinumab had the greatest impact on the cost per PASI 75 differences for both a patient with prior biologic treatment failure $(+\$ 33,819$ to $+\$ 85,763)$ and a patient without prior biologic treatment failure $(+\$ 27,590$ to $+\$ 36,315)$. 
Variations in the PASI 75 efficacy rate of brodalumab and drug cost discount also had relatively large impacts on the cost per PASI 75 differences associated with treatment with ustekinumab vs. brodalumab. Variations in the other parameters had relatively small impacts on the cost per PASI 75 differences. The variation in the cost per PASI 75 difference was on a larger scale for a patient with vs. without prior biologic treatment failure.

In the Monte Carlo multivariable analyses, the mean cost per PASI 75 difference for treatment with ustekinumab vs. brodalumab for a patient with prior biologic treatment failure was $+\$ 54,584(+\$ 32,191$ to $+\$ 86,558)$ and for patient without prior biologic treatment failure it was $+\$ 31,717$ ( $+\$ 26,836$ to $+\$ 37,070)$. For both patients with and without prior biologic treatment failure, in 100\% of the 10,000 random Monte Carlo simulation cycles, ustekinumab

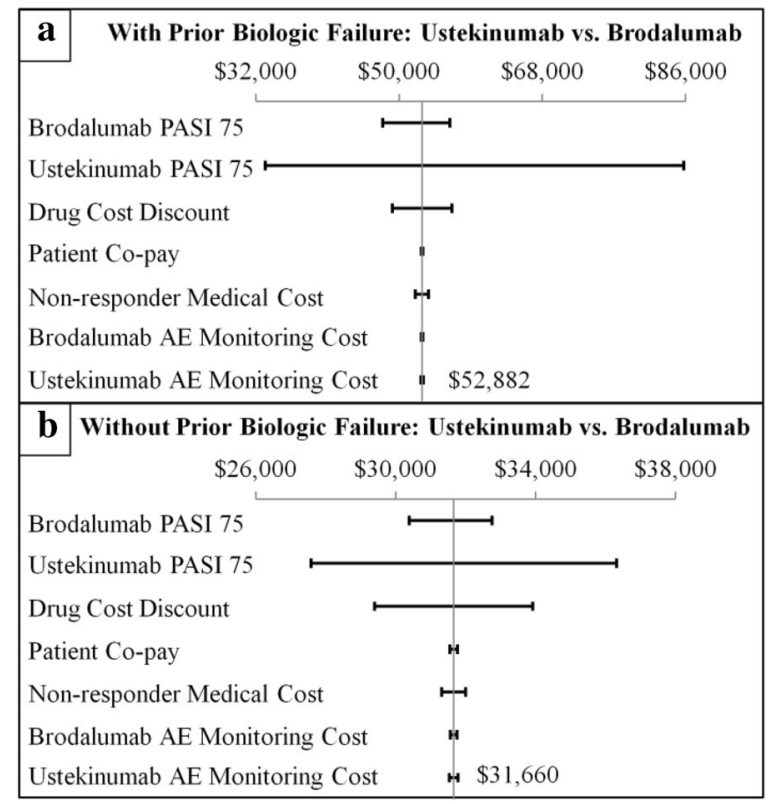

Fig. 5 Univariate sensitivity analysis of the differences in total annual health plan costs per PASI 75 among patients $\mathbf{a}$ with prior biologic failure and $\mathbf{b}$ without prior biologic failure and treated with ustekinumab vs. brodalumab. PASI Psoriasis Area Severity Index. PASI 75 efficacy rates were varied between the ranges of their respective 95\% confidence intervals; incremental cost estimates were varied $\pm 30 \%$. Non-responder medical cost: average annual total medical costs associated with PASI $<75$. $\mathrm{AE}$ adverse event

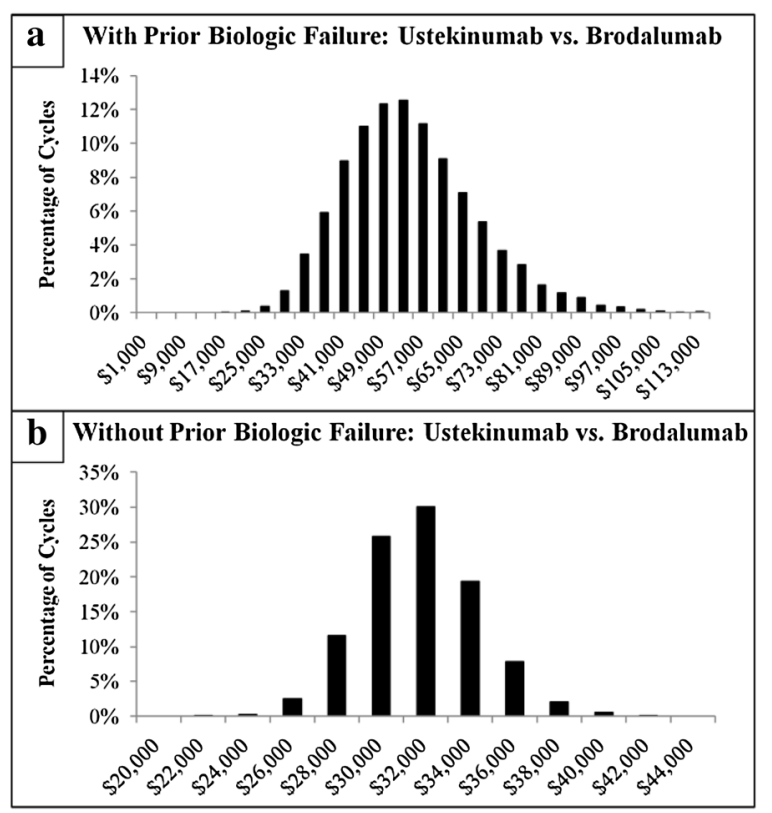

Fig. 6 Multivariable sensitivity analysis of the differences in total annual health plan costs per PASI 75 among patients $\mathbf{a}$ with prior biologic failure and $\mathbf{b}$ without prior biologic failure and treated with ustekinumab vs. brodalumab. PASI Psoriasis Area Severity Index

displayed a higher cost per PASI 75 vs. brodalumab (100.0\% of Monte Carlo simulation cycles had a cost difference $>\$ 0$ ). The mean cost per PASI difference was magnified per PASI 100. In this Monte Carlo multivariable analysis the mean cost per PASI 100 difference was $+\$ 513,666$ $(+\$ 152,129$ to $+\$ 1,875,946)$ for a patient with prior biologic treatment failure and for a patient without prior biologic treatment it was $+\$ 166,954$ ( $+\$ 126,273$ to $+\$ 218,196)$. For both patients with and without prior biologic treatment failure, in 100\% of the 10,000 random Monte Carlo simulation cycles, ustekinumab displayed a higher cost per PASI 100 vs. brodalumab (100.0\% of Monte Carlo simulation cycles had a cost difference $>\$ 0)$.

\section{DISCUSSION}

In the AMAGINE-2/-3 clinical trials, brodalumab exhibited superior efficacy over ustekinumab for the achievement of PASI 75, 90, and 100, regardless of whether moderate-to-severe psoriasis patients were naïve or experienced with biologic 
treatment or were with or without prior biologic treatment failure [10]. When this superior efficacy across these patient types is incorporated into a cost-effectiveness model, as well as the much lower WAC of brodalumab, treatment with brodalumab was associated with substantially better cost-effectiveness ratios (cost per PASI 75, 90, and 100) for patients naïve to and experienced with prior biologic treatment and for patients with and without prior biologic treatment failure. Total annual treatment costs for achievement of PASI 75,90 , and 100 for a patient naïve to prior biologic treatment were $42 \%, 52 \%$, and $64 \%$ respectively, lower per patient treated with brodalumab compared to a patient treated with ustekinumab; for a patient experienced with prior biologic treatment they were $46 \%, 52 \%$, and $70 \%$, respectively, lower; for a patient with prior biologic treatment failure they were $53 \%, 60 \%$, and $75 \%$, respectively, lower; and for a patient without prior biologic treatment failure they were $42 \%, 51 \%$, and $64 \%$, respectively lower. For patients who failed prior biologic treatment ( $40 \%$ of patients in the AMAGINE-2/-3 trials) brodalumab vs. ustekinumab demonstrated 1.5 -fold better efficacy for achievement of PASI 75, 1.8-fold better efficacy for achievement of PASI 90, and threefold better efficacy for achievement of PASI 100 [10]. Thus, the cost-effectiveness of brodalumab vs. ustekinumab was most prominent in the dollar amount for a patient with prior biologic treatment failure, and especially so for achievement of PASI 100 (annual treatment cost difference $\$ 365,606$ ). The results of this economic analysis were robust in that they were consistent with the default findings in both univariate and multivariable sensitivity analyses.

Switching biologic drugs to maintain treatment response is a relatively common practice for patients with moderate-to-severe psoriasis and such switching can impact subsequent biologic drug survival $[15,16]$. Drug survival refers to how long a patient continues with therapy and may be affected by prior treatments, and current treatment efficacy, safety, tolerability, and cost, as well as patient characteristics (e.g., body mass index, disease severity) $[15,16]$. On the basis of the analysis of the AMAGINE-2/-3 clinical trials, brodalumab was more effective than ustekinumab across multiple types of patients with moderate-tosevere psoriasis. Ustekinumab has better drug survival when compared to etanercept, infliximab, and adalimumab $[15,16]$. Further study in the real-world setting is needed to provide insight into the drug survival of brodalumab vs. ustekinumab among moderate-to-severe psoriasis patients initiating first-, second-, and thirdline therapy. This information can then be used to update the expected cost-effectiveness of brodalumab in the real-world setting.

Although the development of targeted biologics has provided patients with much improved management of their moderate-tosevere psoriasis, the substantial cost of biologics can be prohibitive of access to them. A recent study of a small sample of patients with psoriasis $(n=30)$ between January 2011 and October 2016 who received ustekinumab therapy reported that affordability of the biologic drug was the major reason for choosing intermittent, repetitive therapy [17]. Compared with continuous treatment, such a pattern of non-continuous biologic therapy can lead to suboptimal patient outcomes $[17,18]$. It is highly relevant in this cost-conscious era of the US healthcare system to have not only alternative efficacious treatment options but also demonstrate that they are more value-based.

When efficacy data from a large meta-analysis of multiple clinical trials are used [19], brodalumab is less costly than several other biologic drugs-including adalimumab, ixekizumab, secukinumab, and ustekinumab-for achieving PASI 75, 90, and 100 among patients with moderate-to-severe psoriasis [13]. The lower WAC of brodalumab versus the other biologics contributed to these findings [13]. The recently released draft report by the Institute for Clinical and Economic Review on psoriasis treatment states that, of the 11 evaluated drugs for psoriasis treatment, brodalumab is at the lower (more favorable) end of the value spectrum [20]. Furthermore, the draft report concludes that on the basis of their model, initial treatments with apremilast, infliximab, certolizumab, and brodalumab have similar costeffectiveness compared to non-targeted treatment, with brodalumab having the greatest effectiveness [20]. Herein, we further show that 
on the basis of clinical trial efficacy data, brodalumab is expected to have greater cost-effectiveness than ustekinumab across a diverse population of patient types. Although further studies of the efficacy of brodalumab and its drug survival in the real-world setting are warranted, the results of our study may be useful to stakeholders involved in the treatment decision-making processes for patients with moderate-to-severe psoriasis.

\section{Limitations}

PASI efficacy rates were derived from clinical trial data [10]. Although they were based on direct comparison trials, the efficacy rates may be different in real-world settings. Also, the PASI efficacy rates were based on the treatment time frame of 12 weeks and peak efficacy of brodalumab and ustekinumab may differ with longer time frames, especially in the real-world setting. Thus, further study of the efficacy rates of brodalumab and ustekinumab with longer time frames in the real-world setting and follow-up cost analyses are warranted. Other patient outcomes, such as health-related quality of life, long-term cost of adverse events, and potential impact of biologics on the cost of comorbidities of patients with psoriasis were not included in the model and further study is warranted. The average annual total medical costs and AE monitoring costs associated with a non-response to biologic treatment (PASI $<75$ ) were derived from Strand et al. and whether the estimated higher costs for a non-responder in this study are representative of that of moderate-to-severe psoriasis patients treated with brodalumab and ustekinumab in the real-world setting may require further evaluation. However, when these cost parameters were varied in the sensitivity analyses we conducted, we did not find major impacts on the estimated costeffectiveness ratios. Dose escalation, which may be required for patients with moderate-to-severe psoriasis who fail to respond to ustekinumab, was not incorporated into the model and treatment costs for these patients may be much higher than the estimated costs for other patient types. In the prescribing information for brodalumab, dose escalation is not recommended. We used a hypothetical 20\% drug discount for both brodalumab and ustekinumab for a balanced comparison in this regard. This discount and other offered rebates are generally considered proprietary information and may differ in individual health plans. Also, the contracting discount in the current economic model only considered the potential impact of such discount levels on the indication analyzed in the model, moderate-to-severe psoriasis, and was not applied to any other indications. However, this $20 \%$ discount is a commonly used assumption in other similar economic models $[13,21]$. Additionally, we have further varied the drug price by $\pm 30 \%$ range in the one-way sensitivity analyses and the MonteCarlo sensitivity analyses, both of which showed that brodalumab is more cost-effective than ustekinumab when the final drug prices may vary. Lastly, the results of this economic analysis are reflective of biologic drug costs to US commercial payers and may not generalize to other health plan types, such as Medicare or Medicaid.

\section{CONCLUSIONS}

Compared to ustekinumab, treatment with brodalumab was associated with better cost-effectiveness ratios for patients naïve and experienced with biologic treatment and also patients with and without prior biologic treatment failure. The greater cost-effectiveness of brodalumab was most prominent for biologicexperienced and prior biologic treatment failure patients. The expected value of brodalumab estimated in this economic analysis may be useful for the stakeholders involved in the treatment decision-making processes for patients with moderate-to-severe psoriasis.

\section{ACKNOWLEDGEMENTS}

Funding. Sponsorship for this research and article processing charges were funded by Ortho Dermatologics. All authors had full access to all 
of the data in this study and take complete responsibility for the integrity of the data and accuracy of the data analysis.

Authorship. All named authors meet the International Committee of Medical Journal Editors (ICMJE) criteria for authorship for this article, take responsibility for the integrity of the work as a whole, and have given their approval for this version to be published.

Disclosures. SR Feldman served as a consultant for Ortho Dermatologics for conducting this study. SR Feldman has also received speaking, consulting and/or research support from Abbvie, Celgene, Janssen, Lilly and Novartis. $S$ Rastogi is an employee of Ortho Dermatologics. J Lin is an employee of Novosys Health, which has received research funds from Ortho Dermatologics for conducting this study and development of this manuscript.

Compliance with Ethics Guidelines. This article is based on previously conducted studies and does not contain any studies with human participants or animals performed by any of the authors.

Open Access. This article is distributed under the terms of the Creative Commons Attribution-NonCommercial 4.0 International License (http://creativecommons.org/licenses/ by-nc/4.0/), which permits any noncommercial use, distribution, and reproduction in any medium, provided you give appropriate credit to the original author(s) and the source, provide a link to the Creative Commons license, and indicate if changes were made.

\section{REFERENCES}

1. Naldi L, Mercuri SR. Epidemiology of comorbidities in psoriasis. Dermatol Ther. 2010;23:114-8.

2. Kurd SK, Gelfand JM. The prevalence of previously diagnosed and undiagnosed psoriasis in US adults: results from NHANES 2003-2004. J Am Acad Dermatol. 2009;60:218-24.
3. Stern RS, Nijsten T, Feldman SR, et al. Psoriasis is common, carries a substantial burden even when not extensive, and is associated with widespread treatment dissatisfaction. J Investig Dermatol Symp Proc. 2004;9:136-9.

4. Brezinski EA, Dhillon JS, Armstrong AW. Economic burden of psoriasis in the United States: a systematic review. JAMA Dermatol. 2015;151:651-8.

5. Molinelli E, Campanati A, Brsigotti V, et al. Biologic therapy in psoriasis (Part II): efficacy and safety of new treatment targeting IL23/IL-17 pathways. Curr Pharm Biotechnol. 2017;18:964-78.

6. Tausend W, Downing C, Tyring S. Systematic review of interleukin-12, interleukin-17, and interleukin-23 pathway inhibitors for the treatment of moderate-to-severe chronic plaque psoriasis: ustekinumab, briakinumab, tildrakizumab, guselkumab, secukinumab, ixekizumab, and brodalumab. J Cutan Med Surg. 2014;18:156-69.

7. Stelara (ustekinumab). Full Prescribing Information. Janssen Pharmaceutical Companies. 2017. http://www.janssenlabels.com/package-insert/prod uct-monograph/prescribing-information/STELARApi.pdf. Accessed 20 Apr 2018.

8. Siliq (brodalumab). Full Prescribing Information. Valeant Pharmaceuticals. 2017. http://www. valeant.com/Portals/25/Pdf/PI/Siliq-pi.pdf. Accessed 20 Apr 2018.

9. Lebwohl M, Strober B, Menter A, et al. Phase 3 studies comparing brodalumab with ustekinumab in psoriasis. N Engl J Med. 2015;373:1318-28.

10. Papp KA, Gordon KB, Langley RG, et al. Impact of previous biologic use on efficacy and safety of brodalumab and ustekinumab in patients with moderate-to-severe plaque psoriasis: integrated analysis of AMAGINE-2 and AMAGINE-3. Br J Dermatol. 2018. https://doi.org/10.1111/bjd.16464

11. Truven Health Analytics. Red Book. Ann Arbor, MI. http://www.micromedexsolutions.com/micromede x2/librarian/. Accessed 27 Mar 2018.

12. Gu T, Shah N, Deshpande G, et al. Comparing biologic cost per treated patient across indications among adult US managed care patients: a retrospective cohort study. Drugs Real World Outcomes. 2016;3:369-81.

13. Wu JJ, Feldman SR, Rastogi S, et al. Comparison of the cost-effectiveness of biologic drugs used for moderate-to-severe psoriasis treatment in the United States. J Dermatol Treat. 2018;16:1-20.

14. Strand V, Husni E, Griffith J, et al. Economic evaluation of timely versus delayed use of tumor 
necrosis factor inhibitors for treatment of psoriatic arthritis in the US. Rheumatol Ther. 2016;3:305-22.

15. Warren RB, Smith CH, Yiu ZZ, et al. Differential drug survival of biologic therapies for the treatment of psoriasis: a prospective observational cohort study from the British Association of Dermatologists Biologic Interventions Register (BADBIR). J Invest Dermatol. 2015;135:2632-40.

16. Menter A, Papp KA, Gooderham M, et al. Drug survival of biologic therapy in a large, disease-based registry of patients with psoriasis: results from the Psoriasis Longitudinal Assessment and Registry (PSOLAR). J Eur Acad Dermatol Venereol. 2016;30:1148-58.

17. Choi CW, Choi JY, Kim BR, et al. Economic burden can be the major determining factor resulting in short-term intermittent and repetitive ustekinumab treatment for moderate-to-severe psoriasis. Ann Dermatol. 2018;30:179-85.

18. Hjalte F, Steen Carlsson K, Schmitt-Egenolf M. Realworld outcome analysis of continuously and intermittently treated patients with moderate to severe psoriasis after switching to a biologic agent. Dermatology. 2015;230:347-53.

19. de Carvalho AV, Duquia RP, Horta BL, et al. Efficacy of immunobiologic and small molecule inhibitor drugs for psoriasis: a systematic review and metaanalysis of randomized clinical trials. Drugs R D. 2017;17:29-51.

20. Institute for Clinical and Economic Review. Targeted immunomodulators for the treatment of moderate-to-severe plaque psoriasis: effectiveness and value. Psoriasis update: draft evidence report. 27 Apr 2018. Boston, MA. https://icer-review.org/ wp-content/uploads/2017/11/ICER_Psoriasis_Upda te_Draft_Report_04272018.pdf. Accessed 30 Apr 2018.

21. Asche CV, Kim M, Feldman SR, et al. Budget impact model in moderate-to-severe psoriasis vulgaris assessing effects of calcipotriene and betamethasone dipropionate foam on per-patient standard of care costs. J Med Econ. 2017;20:1000-6. 